\title{
Promotion of Exclusive Breastfeeding in a Clinic Setting Did Not Increase Its Rates
}

\author{
Allison L. Scott, DNP, RN, IBCLC ${ }^{1}$ \\ Marie-Rachelle Narcisse, PhD, CHCQM ${ }^{2}$ \\ Lyla Lindholm, DNP, RN, CNS ${ }^{3}$
}

Share this:

\begin{abstract}
Breastfeeding is known to have many protective effects on childhood health. Breastfeeding without supplementation or complementary foods until 6 months of age is inversely related to several health problems, including childhood obesity. Because of the known beneficial effects on infant health of breastfeeding, education on breastfeeding may impact exclusive breastfeeding rates, resulting in improved infant health status. This retrospective study, using Pender's health promotion model and the theory of planned behavior (Pender, Murdaugh, Eु Parsons, 2011), explored the effect of a breastfeeding education intervention for quality improvement in an ambulatory care clinic on the rate of exclusive breastfeeding during the first 4 months of infancy, or short-term exclusive breastfeeding. Exclusive breastfeeding rates were measured after the 4-month well exam, which represents the time of the existence of the quality improvement intervention for 4-5 months. The expected outcome was a minimum 15\% increase in exclusive breastfeeding rates in the participants who received the educational program as compared to the individuals who did not receive the breastfeeding education intervention. Contrary to expectations, the group receiving standard care had significantly higher rates of exclusive breastfeeding than those receiving the intervention.
\end{abstract}

Keywords: breastfeeding, breastfeeding education, exclusive breastfeeding, complementary feeding

Breastfeeding has significant health benefits for mothers and infants. The American Academy of Pediatrics (AAP, 2012) recommends exclusive breastfeeding for the first 6 months of life, and continued breastfeeding for at least 1 year. Breast milk's protective effect increases with duration and exclusivity of breastfeeding (Matias, Nommsen-Rivers, \& Dewey, 2012). Exclusive breastfeeding versus breastfeeding with complementary foods appears to have a more significant effect in decreasing the risk of a child becoming overweight (Centers for Disease Control and Prevention [CDC], 2007).

The AAP priority of exclusive breastfeeding until at least 4 months of age is a national priority for improving children's health and reducing childhood obesity (Dolinsky, Siega-Riz, Perrin, \& Armstrong, 2011).

Exclusive breastfeeding is the reference or normative model against which all alternative feeding methods must be measured with regard to growth, health, development, and all other short-and long-term outcomes. (U.S. Department of Health and Human Services [USDHHS], 2011, p. 5)

\footnotetext{
1.Als002@uark.edu

2.narcisse@uark.edu

3. 1indholml@umkc.edu
}

Stein and Kuhn (2009) state that new studies have confirmed breastfeeding benefits for the mother-baby dyad and reinforce emphasis on exclusive breastfeeding for the first 6 months of life. Longer duration of breastfeeding has been associated with decreased rates of childhood obesity (Snethen, Hewitt, \& Goretzke, 2007). Breastfeeding for 9 months can reduce the odds of becoming overweight by more than 30\% (CDC, 2011c).

Healthy People 2020 set a national goal of exclusive breastfeeding through 3 months of life at $46.2 \%$ and through 6 months of life at $25.5 \%$. Current national exclusive breastfeeding rates are $35 \%$ at 3 months and $14.8 \%$ at 6 months (CDC, 2011a). Despite the inordinate amount of evidence about the benefits of breastfeeding, only one state (Vermont) meets the six breastfeeding outcome indicators with 40 of 50 states meeting none (CDC, 2011a). An additional goal for Healthy People 2020 is to reduce the proportion of breastfed newborns receiving formula supplementation within the first 2 days of life to $14.2 \%$ from the baseline of $24.2 \%$ measured in 2006.

According to the CDC (2011b), low rates of breastfeeding add $\$ 2.2$ billion a year to medical costs. Bartick and Reinhold (2010) estimate a $\$ 13$ billion savings per year nationally if $90 \%$ of American families breastfed exclusively 


\section{The Importance of Breastfeeding Education for Nurses}

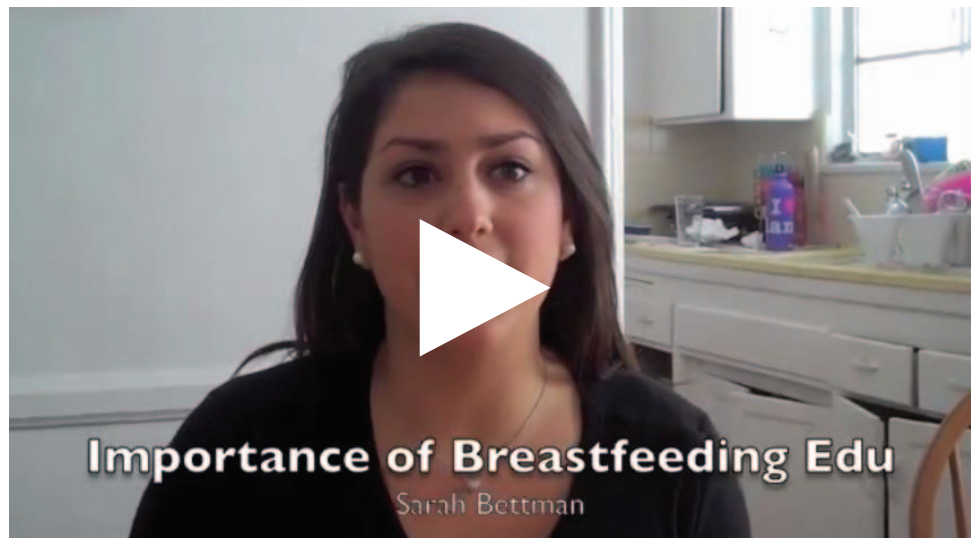

https://www.youtube.com/watch?v5TbZbwVs9teg

for 6 months. Breastfeeding promotion can affect morbidity and quality of life because evidence supports a positive effect on breastfeeding initiation and duration.

Early introduction of formula and discontinuation of breastfeeding raises the risk of obesity, diabetes, respiratory infections, and sudden infant death syndrome (CDC, 2011b). Policy level changes for obesity prevention have been recommended by the CDC, with a strategy to encourage breastfeeding as 1 of 24 strategies described by an expert panel (CDC, 2011b, p. 1). Because of health benefits of breastfeeding, healthcare professionals are strongly encouraged to support breastfeeding exclusively for 6 months and continue with complementary foods for a year or more (AAP, 2012; Institute of Medicine, 2011).

\section{Purpose}

The purpose of this study was to determine if breastfeeding education with mothers during the postnatal period, regarding the benefits of exclusive breastfeeding, would increase the rate of exclusive breastfeeding at four months of age. Specific emphasis on the protective factor of exclusive breastfeeding during infancy on childhood obesity was included in the education. It was hypothesized that a focused educational session during each well-baby visit in an ambulatory care center, coupled with phone-call support by a pediatric nurse practitioner/International Board Certified Lactation Consultant (PNP/IBCLC), would positively impact exclusive breastfeeding rates at 4 months of age.

\section{Method}

Through a retrospective medical record review, this study compared a control group, receiving standard feeding education from providers who are not certified in lactation education and no scheduled phone follow-up, to an intervention group receiving newly implemented education specific to benefits of exclusive breastfeeding and minimum of one follow-up phone consultation from a nurse practitioner/IBCLC. Primary outcome measurement of exclusive breastfeeding was determined through a chart review after implementation of exclusive breastfeeding education by the PNP/IBCLC for a minimum of 4 months. Two-month exclusive breastfeeding rates were also tracked through a chart review.

\section{Sample and Setting}

This study population included all breastfeeding motherbaby dyads seen at an ambulatory care pediatric clinic by the 7th day of age. The study setting employs 18 physicians and 2 advanced practice nurses (APNs). The investigator is employed as an APN/PNP/lactation consultant at the study site and has been an IBCLC since 1989 .

Study participants spoke English or Spanish as their primary language and were minimum of 37 weeks gestation at birth, had no neonatal intensive care stay, no treatment for hyperbilirubinemia, and no illness that would contraindicate breastfeeding. All breastfeeding mother-baby dyads meeting inclusion criteria and seen by the PNP/IBCLC received the education intervention, focusing on exclusive breastfeeding beginning with their initial well-baby visit. Mothers seen by another provider within this clinic received the prior existing education on breastfeeding and were not seen by the PNP/IBCLC. The sample included 21 participants in the intervention group and 22 participants in the control group. 


\section{Educational Intervention}

Exclusive breastfeeding education, defined as written and verbal education provided by the PNP/IBCLC, was given at the initial visit, 2-week visit, and 2-month visit. Educational counseling provided by the PNP/ IBCLC was given during each 20-30 minute clinic visit. Breastfeeding education regarding benefits of exclusive breastfeeding, including decreased childhood obesity, was addressed at each visit. The International Lactation Consultant Association's (ILCA) Inside Track handouts on exclusive breastfeeding, feeding solids, and breastfeeding and returning to work were given at the initial, 2-week, and 2-month well visit, respectively. The initial visit also included a handout developed by the PNP/LC using Guide to Breastfeeding Interventions (CDC, 2009). All handouts were available in English and Spanish. One follow-up phone call between the 2-week and 2-month visit assessed for any breastfeeding barriers and allowed reinforcement of the benefits of exclusive breastfeeding.

The mothers, seen by another APN or physician not trained in lactation, received existing standard feeding assessment and education. All breastfeeding mothers had access to the PNP/IBCLC, if desired, for lactation difficulty, but did not receive specific education regarding exclusive breastfeeding benefits.

\section{Results}

Descriptive analysis was used to calculate the number and percentage for each categorical variable and to determine mean and standard deviation for each sociodemographic variable. The number of exclusively breastfed infants at 2 months and 4 months was compared with the intervention group that received the exclusive breastfeeding education, and with the control group that did not receive the newly implemented exclusive breastfeeding education.

Analysis of data revealed no significant differences among the group receiving the quality improvement initiative and the group receiving the standard breastfeeding education regarding delivery type, previous breastfeeding experience, ethnicity, and smoking. Ethnicity of the intervention group was $71 \%$ White and $29 \%$ Hispanic. Small differences were observed in the maternal education under 12 years $(29 \%$ intervention and $22 \%$ control). The median maternal age was 29 years for the intervention group and 28 years for the control group. Eight subjects were lost in the intervention group because of lack of follow-up with scheduled appointments, resulting in 13 charts for full review. All 22 subjects were included in the control group.
Contrary to expectations, at 4 months, $31 \%$ of the intervention group was exclusively breastfeeding compared to $55 \%$ of the control group. In addition, $8 \%$ of the intervention group were complementary feeding compared to $14 \%$ of the control group.

\section{Discussion}

Exclusive breastfeeding rates were higher in the group receiving the standard clinic breastfeeding education and support compared with those who received the quality improvement breastfeeding education. This finding is in disagreement with a study of more than 700 infants, recently published, evaluating establishment of an improved office protocol to promote exclusive breastfeeding (Corriveau, Drake, Kellams, \& Rovnyak, 2013).

Several limitations of the study exist and results were likely affected by (a) difficulty with scheduling consistent well visits with the PNP/IBCLC, (b) difficulty completing the scheduled phone follow-up prior to 2 months, and (c) small sample size. This study was limited by the confounding variables not addressed in the study, specifically insurance or Medicaid status and breastfeeding support in the home. Corriveau et al. (2013) found insurance status to be highly significant, with odds of exclusive breastfeeding for those receiving Medicaid to be $28 \%$ versus $47 \%$ odds for those with private insurance. Although maternal education was assessed, insurance status was not and could be an important socioeconomic factor influencing breastfeeding rates.

\section{Conclusions}

The results from this study suggest that additional education specific to benefits of exclusive breastfeeding does not increase rates of breastfeeding at 2 and 4 months of age. The findings from this study are in contrast to those of a much larger study that did confirm additional education, involving prenatal, hospital, and clinic personnel, resulted in increased exclusive breastfeeding rates up to 6 months of age (Corriveau et al., 2013). Use of standardized education materials and increased lactation support can impact exclusive breastfeeding rates, but the influence may be minimal or nonexistent unless a combination of prenatal, immediate postnatal, and clinic follow-up, involving all healthcare providers, is implemented.

This study serves as an evaluation of a small-scale quality improvement, using a nurse/lactation consultant, on exclusive breastfeeding rates in a clinic setting among primarily White and Hispanic mothers. Further studies should explore the impact of a clinic education protocol involving all providers and collaboration with lactation personnel in the hospital setting. 
To address the public health initiative of increasing breastfeeding rates and impacting childhood obesity at the beginning of life, further studies of education and lactation support must be completed. To meet Healthy People 2020 goals (USDHHS, 2010), research must continue to determine what determines mothers' choice to breastfeed and continue breastfeeding through the first 6 months of their infant's life.

\section{References}

American Academy of Pediatrics. (2012a). Breastfeeding and the use of human milk. Pediatrics, 129(3), e827-e841. http:// dx.doi.org/10.1542/peds.2011-3552)

American Academy of Pediatrics. (2012b). Where we stand on breastfeeding. Retrieved from http://www.healthychildren.org/English/agesstages/baby/Breastfeeding/Pages/Where-We-Stand-Breastfeeding

Bartick, M., \& Reinhold, A. (2010). The burden of suboptimal breastfeeding in the United States: A pediatric cost analysis. Pediatrics, 125(5), e1048-1056. http://dx.doi.org/10.1542/ peds.2009-1616

Centers for Disease Control and Prevention. (2007). Does breastfeeding reduce the risk of pediatric overweight? Atlanta, GA: Centers for Disease Control and Prevention.

Centers for Disease Control and Prevention. (2009). The CDC guide to breastfeeding interventions: Educating mothers. Retrieved from http://www.cdc.gov/breastfeeding/resources/guide.htm

Centers for Disease Control and Prevention. (2011a). Breastfeeding report card-United States, 2011. Retrieved from http://www.cdc gov/breastfeeding/data/

Centers for Disease Control and Prevention. (2011b). CDC reports that preventing childhood obesity begins in hospitals. Clinical Lactation, 2(4), 39.
Centers for Disease Control and Prevention. (2011c). Vital signs: Hospital support for breastfeeding. Preventing obesity begins in hospitals. Retrieved from http://www.cdc.gov/vitalsigns/ Breastfeeding/index.html

Corriveau, S. K., Drake, E. E., Kellams, A. L., \& Rovnyak, V. G. (2013). Evaluation of an office protocol to increase exclusivity of breastfeeding. Pediatrics, 131(5), 942-950. Retrieved from http://pediatrics.aapublications.org/content/ early/2013/03/27/peds.2012-1310

Dolinsky, D. H., Siegariz, A. M., Perrin, E., \& Armstrong, S. C. (2011). Recognizing and preventing childhood obesity. Contemporary Pediatrics, 28(1), 32-42.

Institute of Medicine. (2011). Report at a glance: Early childhood obesity prevention policies. Retrieved from http://www.iom.edu/ Reports/2011/Early-Childhood-Obesity-Prevention-Policies/ Report-Brief.aspx

Matias, S. L., Nommsen-Rivers, L. A., \& Dewey, K. G. (2012). Determinants of exclusive breastfeeding in a cohort of primiparous periurban Peruvian mothers. Journal of Human Lactation, 28(1), 45-54.

Pender, N., Murdaugh, C., \& Parsons, M. A. (2011). Health promotion in nursing practice (6th ed.). Upper Saddle River, NJ: Pearson Education.

Snethen, J. A., Hewitt, J., \& Goretzke, M. (2007). Childhood overweight: The infancy connection. Journal of Obstetric, Gynecologic, E̊ Neonatal Nursing, 36(5), 501-510.

Stein, Z., \& Kuhn, L. (2009). Breast feeding: A time to craft new policies. Journal of Public Health Policy, 30(3), 300-310.

U.S. Department of Health and Human Services. (2010). Healthy People 2020. Retrieved from http://www.healthypeople.gov/

U.S. Department of Health and Human Services. (2011). Executive summary: Surgeon general's call to action to support breastfeeding. Retrieved from http://www.surgeongeneral.gov/topics/ breastfeeding/executivesummary.pdf

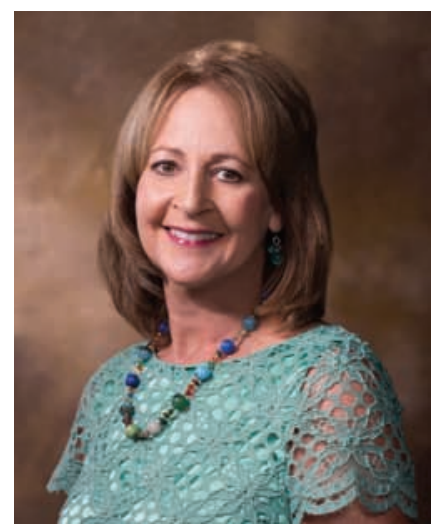

Allison Scott, DNP, RN, IBCLC, is a certified lactation consultant and Pediatric Nurse Practitioner. For the past 25 years, she has worked with breastfeeding mothers in hospital and clinic settings. Allison currently functions as the lactation consultant in a large pediatric practice. She is an associate professor at University of Arkansas, Fayetteville, in the BSN program, teaching pediatric nursing. Her research interests focus on breastfeeding education and prevention of childhood obesity. 

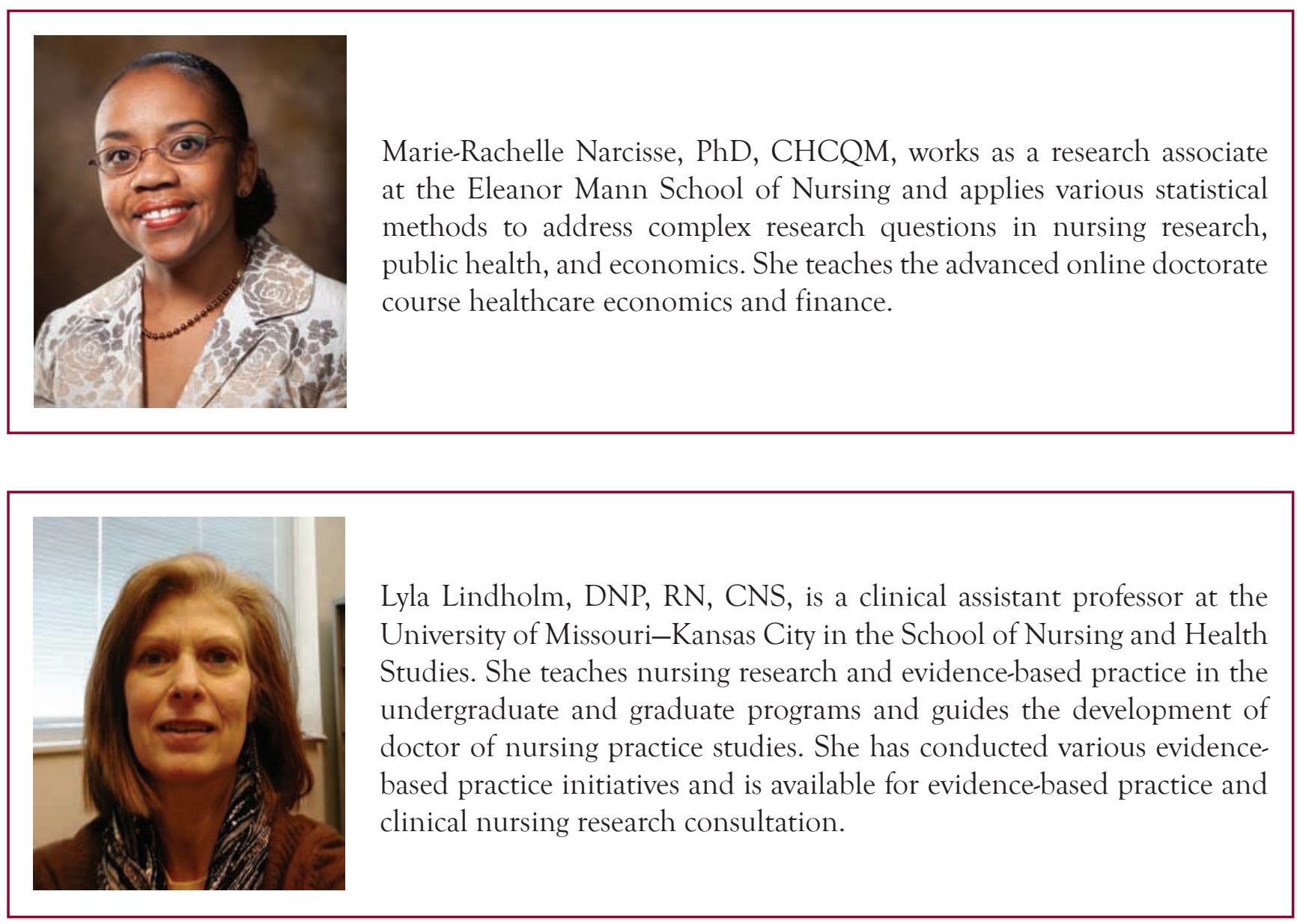

\section{Hospital Breastfeeding Report Card From California WIC}

The California WIC Association and UC Davis Human Lactation Center have published Bringing Breastfeeding Home: Building Communities of Care, a policy update on California breastfeeding and hospital performance. State and county fact sheets provide specific data, including exclusive breastfeeding rates for each hospital in individual counties, and comparing California counties to each other. The report documents that hospitals have dramatically increased their breastfeeding rates, resulting in a significant statewide increase since 2010.

Source: USBC

\section{Working Families Action Memo From Center for American Progress}

The Center for American Progress has published the Working Families Action Memo, providing background information, tools, and resources for individuals and organizations to build support in their communities on key policy priorities for working families.

Source: USBC 